\title{
An Innovative Framework for Dynamic Traffic Lights Management Based on the Combined Use of Fuzzy Logic and Several Network Architectures
}

\author{
Fabio Arena (iD, ${ }^{1}$ Giovanni Pau $\left(\mathbb{D},{ }^{1}\right.$ Anca Ralescu $\left(\mathbb{D},{ }^{2}\right.$ Alessandro Severino $\left(\mathbb{D},{ }^{3}\right.$ \\ and Ilsun You ${ }^{4}$ \\ ${ }^{1}$ Faculty of Engineering and Architecture, Kore University of Enna, Enna 94100, Italy \\ ${ }^{2}$ College of Engineering and Applied Science, University of Cincinnati, 2901 Woodside Drive, Cincinnati, OH 45221, USA \\ ${ }^{3}$ Department of Civil Engineering and Architecture, University of Catania, Catania 95123, Italy \\ ${ }^{4}$ Department of Information Security Engineering, Cryptology and Mathematics, Kookmin University, \\ Seoul 02707, Republic of Korea
}

Correspondence should be addressed to Alessandro Severino; alessandro.severino@unict.it

Received 2 December 2021; Revised 18 January 2022; Accepted 27 January 2022; Published 23 February 2022

Academic Editor: Socrates Basbas

Copyright $\odot 2022$ Fabio Arena et al. This is an open access article distributed under the Creative Commons Attribution License, which permits unrestricted use, distribution, and reproduction in any medium, provided the original work is properly cited.

\begin{abstract}
The development of Information and Communication Technologies (ICT) has now reached some entirely unexpected domains. Many applications in modern cities lead to novelties, resulting in new habits of the citizens of tomorrow's smart cities. These innovations include Intelligent Transportation Systems (ITS). An important application domain of ITS is undoubtedly represented by the dynamic and optimized management of traffic-lighted road intersections. Although several works have already been presented in the literature over the years, many have not considered the new perspectives regarding the source of data to manage traffic lights, i.e., not only Wireless Sensor Networks (WSNs), but, mainly, the vehicular communications. This paper introduces an innovative approach to dynamically regulating traffic light cycles and phases in an isolated intersection. The suggested method can fit data management from WSNs and vehicular communications through IEEE 802.11p and LTE-V2V, employing various Fuzzy Logic Controllers (FLCs) that manage vehicles turning movements for dynamic controls of both the phase and the green time of traffic lights. The results obtained will allow us to observe that the proposed application is better than the others. Each of the implemented configurations will bring advantages and disadvantages that allow choosing one configuration or another based on specific project requirements.
\end{abstract}

\section{Introduction}

Many countries are experiencing a rapid increase in the growth rate of their urban areas, caused by people's migration to cities in search for education, better jobs, and other social and cultural amenities. Currently, more than $50 \%$ of the world's population live in cities. For this reason, city infrastructures must continuously adapt to new technological opportunities, to maintain a high quality of life and services for their inhabitants. The increasing interactivity and technological advances gave rise to the new concept of smart city, which identifies a series of areas of development that transform the essence and functionality of the modern cities. These areas range from smart mobility to the smart economy, intersecting different areas and development themes. In recent years, intelligent mobility has enjoyed increasing interest in the scientific community, to the point that it has become the subject of numerous applications that only a few years ago could only be imagined [1]. In Figure 1, it is possible to observe an example of integration of different types of vehicles (smart mobility) within a modern smart city.

In parallel, Information and Communication Technologies (ICT) profoundly change people's lifestyles, transform many daily habits, and transform the very structure of modern cities. The integration of information 


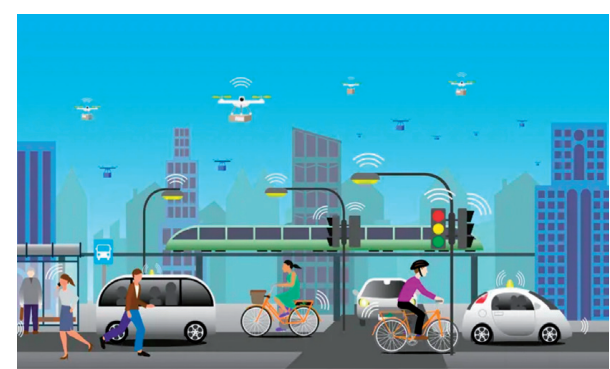

FIgURE 1: An example of smart mobility [2].

technologies in the transport area has led to Intelligent Transportation Systems (ITS). This development area is currently playing a vital role in integrating new transport paradigms, which allow delivering new services to endusers, providing them with integrated tools. These services are complemented by the smart cities with some features that allow the integration of ICT and ITS, thus offering a series of new opportunities to the end-users and the citizens.

The dynamic management of traffic-lighted intersections represents a research segment of great interest in the scientific community, but it is probably even less thorough. In particular, many scientific articles in the literature deal with static applications, that is, through the fixed implementation of sensors or sensor networks (typically located at the side of the road) designed to detect the presence or absence of vehicles at a given time of day. This data is then managed by network infrastructures that control the ignition and duration of the different colors of the traffic lights. Although static sensor based technologies are effective for the management of traffic light systems, they are outdated when it comes to managing the entire road network dynamically [3].

This paper focuses on a particular perspective related to the smart city, i.e., smart mobility, presenting a solution for an automated and effective management of traffic-lighted intersections. This innovative work in the area of dynamic control at road intersections traffic adapts in real-time and dynamically the cycles and phases of the traffic lights without the deployment of sensors along the roadway. The proposed approach manages real-time data through modern technologies for vehicular communication, including Vehicleto-Vehicle (V2V) and Vehicle-to-Everything (V2X), assisted by Fuzzy Logic Controllers (FLCs).

From this point on, this paper is organized as follows. Section 2 analyzes the state of the art in the literature. This analysis represents a crucial starting point for reflection, which led to the investigation of areas that have not yet been addressed. Section 3 introduces the proposed approach. The advantages of an intelligent traffic light system are evaluated, and a new implementation solution is introduced. The proposed approach is presented at a high level of abstraction, allowing for use inside any upstream network infrastructure (for instance, cellular, wireless, or otherwise). Next, the performance evaluations of three different network construction hypotheses are analyzed in Section 4 and highlight all the advantages introduced by the adoption of an intelligent traffic light system to the point that any previous design hypothesis is highly obsolete. Finally, Section 5 concludes the paper by providing some thoughts for future insights.

\section{The Management of an Intelligent Traffic Light System: Analysis of Cases in the Literature}

Road intersections, that is, the places where two or more road meet, are important elements for the management of a traffic light system. In specific scenarios, the trajectories traveled by pedestrians and/or vehicles can intersect, creating conflict points that produce traffic obstructions and present safety issues for the entire road network [3].

The number of conflict points increases with the number of roads (and road lanes) that intersect. The main aim of traffic lights regulation is the increase of capacity and decrease of delays, together with the increase of traffic safety. Moreover, the intelligent management of traffic lights also makes it possible to the following:

(i) Reduce average waiting times at intersections

(ii) Avoid the onset of congestion, thus reducing the length of the queues

(iii) Decrease fuel consumption and therefore reduce atmospheric pollution produced by exhaust gases

(iv) Guarantee specific time slots in which it is possible to clear the intersection without significant dangers

In intersections regulated with traffic lights, the points of conflict are resolved by assigning a specific time interval to each flow of traffic in which the crossing of the conflict area is allowed. The division of time is obtained with the use of light signals required by specific legislation. The traffic light regulation is based on a universally recognized convention according to which a traffic light can have only three colors, with the light indicators activated in a precisely ordered succession: green (for go-ahead), yellow (for stop warning), and red (for stop immediately/do not drive). The configuration of this chromatic characterization, used in most countries of the world, consists of constant time intervals for switching between the three colored lights.

In general, it is possible to distinguish the traffic lights into three types based on the number of phases or 'times': those with three times, those with four, and finally those with five. This paper will analyze the three-time traffic light, the type of traffic light most present in Italy and throughout Mediterranean Europe and in the United States, South America, Japan, and China. This type of traffic light is widely used because it is easy to understand and practice. Therefore, the sequence of colors will always be the same: green, yellow, and red.

While the usefulness of the traffic light system for road safety purposes is unmistakable, to avoid collateral weaknesses, a careful study of appropriate technical specifications is necessary. One of the most important specifications is the timing of the various phases and traffic light cycles. A traffic light cycle consists of sequence of traffic light indications at 
the end of which the exact configuration of lights existing at the beginning of the sequence itself is repeated. A phase is the part of the cycle in which green is simultaneously assigned to a set of mutually compatible traffic currents (i.e., which do not create points of conflict). A phase is complete if it is impossible to add different currents compatible with those already included [4].

Adjusting the duration of the cycle and duration of the phases with extreme accuracy avoids the onset of queues and, accordingly, traffic congestion. The duration of the green time and the complete traffic light cycle must be suitably calibrated regarding a specific traffic situation. The design of specific traffic light plans can be implemented based on the observation of at least four different situations:

(i) The peak of the traffic in the morning

(ii) The peak of the traffic in the evening

(iii) Traffic in the other hours of the morning

(iv) Traffic in the other hours of the evening

This is a rough design that must take into account several parameters. Typically, a complete traffic light cycle can last 84,94 , or 120 seconds. Its regulation must be defined according to the expected traffic flow (the more significant the expected traffic, the shorter the duration of a traffic light cycle). As a result, within a complete traffic light cycle, all the lanes involved in the intersection will have a constant green, yellow, and red time throughout the day.

It must be noted that each phase must have a minimum green duration of 10 seconds for any vehicular current. On the other hand, regarding the maximum duration of the red, it depends on many factors. For pedestrians and cyclists, the maximum threshold of 60 seconds should not be exceeded, while, for vehicular traffic, a duration as big as double can be accepted. Finally, as regards the duration of the yellow light (usually from 3 to 5 seconds), it is established based on the expected average speed of the cars that intend to cross the intersection, and the higher the speed, the longer the duration of the yellow light. In particular,

(i) If there is an intersection where the average speed of the vehicles is equal to $50 \mathrm{Km} / \mathrm{h}$, then the yellow signal will last 3 seconds

(ii) If, instead, the average speed of the vehicles is equal to $60 \mathrm{Km} / \mathrm{h}$, then the yellow signal will last 4 seconds

(iii) Finally, if the average speed of the vehicles will be equal to $70 \mathrm{~km} / \mathrm{h}$, then the yellow will last for 5 seconds

An alternative approach to the static management of traffic light systems can be achieved employing specific techniques such as the automated regulation of the different phases of an intelligent traffic light. Some proposals already present in the literature are very diversified. Real-time traffic information is collected from various sources such as cameras, wireless sensors, or wireless sensor networks (WSN) placed along the roads and controlled by precise controllers, radio-frequency identification (RFID) devices, accessories with vehicular ad-hoc network (VANET) technology for vehicle-to-vehicle (V2V) communications or vehicle-to-infrastructure (V2I), and approaches that introduce adaptive algorithms for traffic light control and regulate both the sequence of phases and their duration. Some approaches exploit multiple technologies at the same time in order to be able to employ all their advantages.

Information from video cameras is exploited in some works, e.g., $[1,3]$, where an estimate of the traffic density and the classification of vehicles with video monitoring systems are also acquired. In [3], the authors focus mainly on the use of algorithms that manage the switching of traffic lights based on the density of vehicles in the queue, thus aiming to achieve several results including the reduction of the (1) traffic congestion on the roads, (2) frequency of road accidents, (3) fuel consumption, and (4) average waiting times in queues.

The approach proposed in [4] does not use the same technology as $[1,3]$, which do not provide any tolerance for the failure of a part of the video monitoring network. That is, for an intersection with $n$ roads, and using as many $n$ video cameras, the failure of any of these camera would lead to an excessively low service level. Instead, in [4], the proposed system guarantees a high tolerance to errors such as failure of a sensor in the WSN, and the system would present a very insignificant lowering of the service level. Moreover, the ease of installation favored by a WSN would involve a quick replacement of any sensor. A certain degree of redundancy of the sensors present in the network minimizes any partial failures of the detection system. Furthermore, from implementation point of view, the system proposed in [4] is simple with small data exchanges in real-time, which determines a very low computational cost.

Another type of approach involves the use of RFID technologies. This approach does not need to know the type of vehicle passing through since all vehicles have the same priority. The use of RFID devices proposed in [5], which also introduces a self-organized traffic control scheme that helps facilitate emergency response operations, allows covering small areas (short-range function). Therefore, in the case of nonnegligible queues, the green signal would not be set adequately in a short time.

On the other hand, other approaches implement the IEEE 802.15.4 communication protocol, whose communication ranges (between the various wireless devices) vary as the transmission power varies and consequently guarantees greater flexibility and scalability. In these cases, it is fast and straightforward to implement suitable devices that also consider the circumstances in which any emergency vehicles are present in the carriageways. It can be assigned the green signal with priority.

The algorithms proposed in [6] consider various traffic factors, such as traffic volume, waiting times, and vehicle density, to determine the optimal duration of the green time. The algorithms select the phase with the highest execution priority through a simple IF-THEN construct and calculate the duration of the green time as a "crisp"/exact value. Although these algorithms are fascinating and present good simulation results, they nevertheless meet with significant weaknesses when implemented real situations. In this sense, it must be observed that this design solution does not specify 
the wireless communication protocol used. There is no indication of the computational cost of the algorithms presented. This condition means that if the computational cost of these algorithms is very high, the generation of the green time would require an extensive time interval, thus making the data obtained already obsolete with a consequent decrease in the level of service. Also, in [6], it can be recognized that simple rules manage traffic flows. On the contrary, the solution proposed in [4] is more suitable for the design of a traffic sign control system, thanks to its ability to manage the uncertainties associated with the input and output variables (for instance, the number of vehicles in the queue, the phase to be managed and the green time of the traffic lights).

Traffic control systems are large nonlinear stochastic systems, and as a result, finding the optimal traffic signal settings is extremely challenging. Consequently, numerous works reported in the literature propose traffic control systems based on Computational Intelligence (CI), in particular, fuzzy logic controllers, which are suitable for managing traffic lights, as their operating principle is inspired by the way humans reason. Hence, these controllers make decisions based on direct experience gained in the specific environment considered. They learn through specific inference rules that incorporate human feelings on the traffic light system and reflect the human reaction to the system's behavior. In control systems such as those implemented in [7], only variables indicating the traffic of the current phase are taken into consideration, without considering the length of the queues of the other phases.

Numerous applications use WSN for traffic management. One of the most popular approaches uses VANET. All the vehicles involved are exploited in these applications as if they were wireless routers or mobile nodes of the network itself. This situation allows vehicles to connect and create a network. The approach proposed in [8] uses a VANET to collect and aggregate speed and position information relating to individual vehicles in real-time to optimize signal control near a road intersection.

On the other hand, in [9], the authors exploited the V2V and V2I communications typical of VANET networks to propose distributed algorithms to improve traffic flow in real time. However, this particular approach will have to consider some problems related to the mobility and stability of the system. This issue happens because the number of vehicles, in the various hours of the day, is subject to sudden random changes with consequent continuous and uninterrupted variation of the hosts entering and leaving the network. In critical situations of a high density of vehicles present in the carriageways, the VANET would risk saturation, with the consequent collapse or, at best, through the use of adequate controls carried out on congestion, many vehicles would not be accepted and inserted into the network.

The hypothesis developed by the authors of [10] is provided for the use of a fuzzy logic controller for each traffic light phase, thus implementing four inputs and a single output. These four inputs allow receiving the data collected from the four carriageways (always assuming that the considered context deals with a 4 -way intersection). The fuzzy logic controller will choose the highest "alarm" value to be taken into consideration and then output the enabling signal to the road that has been chosen to have green. In this way, the most congested road will have priority, and the goahead signal (green traffic light) will be attributed to it. Although this type of approach turns out to be a good starting point, this application of the fuzzy logic controller does not guarantee an optimal service. Suppose that there is a very high traffic flow on one of the four roads, while, in the remaining three roads, few vehicles are presented per minute. In this case, vehicles belonging to low traffic roads will have very long waiting times, thus obtaining a type $\mathrm{F}$ service level (absolutely inadequate). This limit could be exceeded by also providing a parameter that determines the waiting time in the queue: the higher this parameter is, the more the alarm relating to the road concerned will increase.

The authors of [11] developed a comprehensive data collection system at the University of Manitoba, Winnipeg, MB, Canada. The developed system employs various wireless network technologies and devices to collect traffic data from an intersection along the main road within an urban context. A Bluetooth device detects the vehicle's closeness and the information on the vehicle's trajectory. The data collected is transmitted to the master node via the IEEE 802.15.4 (ZigBee) protocol. The central device manages all incoming data for real-time information about the intersection and sends the data to the server every five minutes via GSM cellular communication. The server stores the information in the database accessed by the consumer and corporate websites to get the correct data in real-time. However, although the structure is well organized, this approach is neither very flexible nor straightforward in its implementation. These problems could be overcome thanks to the use of multiple homogeneous networks, which use simple and flexible communication protocols.

All the approaches analyzed so far present some weaknesses. Firstly, they are obsolete and unsuitable for a truly dynamic management of traffic-lighted intersections. Secondly, the technologies seen so far do not take advantage of vehicular communications and the increasing spread of intelligent vehicles on roads (e.g., driverless vehicles, although their presence on roads is still minimal). However, vehicles with a certain degree of range (at least level 2 or 3 of autonomous driving levels) are increasingly common. The approach proposed in this paper seeks to provide a new key contribution precisely in the direction of these new technologies, experimenting with implementing a network infrastructure that exploits vehicular communications.

\section{The Proposed Experimental Approach}

The analysis carried out in the previous section highlights that the most suitable solution for highly variable traffic flows over time is the one that provides a fully implemented traffic light control: for instance, the traffic-lighted systems in which the green times are determined according to the dynamic characteristics of the traffic $[12,13]$. 
These implemented systems do not act upstream. Therefore, the beginning and the duration of the green times are set adaptively, based on the presence and movement of individual users, in each specific scenario. Fully implemented control requires more expensive equipment and a more significant amount of work in terms of design than semi-implemented and fixed-time control cases. Hence, the concept of complete implementation is more efficient when the intersection to be controlled is subject to traffic that exhibits highly variable behaviors over time [14, 15]. More precisely, advantages of this particular type of approach include the following:

(i) Traffic situations that arise during the day are many and very different from each other; this situation makes it easy to establish that it is not possible to treat them all in the same way, with a relative number of plans at fixed times; adaptability is one of the significant advantages brought about by a fully implemented approach;

(ii) It improves coordination with adjacent installations when the geometric situation of coordination is unfavorable;

(iii) It reduces the average waiting times of a vehicle in a queue and, consequently, reduces the emissions of harmful gases.

The design hypothesis, the subject of in-depth analysis of this paper concerns the automated management of an intelligent traffic light system. According to this hypothesis, it can be assumed that the solution is placed near a four-way road intersection. Among the initial hypotheses, it is also possible to imagine a certain number of vehicles in all available directions and that the traffic conditions are intermediate concerning any peak situations. The latter usually occurs at particular times of the day, which typically match with the early morning hours, at lunchtime, and, finally, at the early evening hours (late afternoon).

Compared to the cases analyzed in the literature, the proposed approach provides a fundamental innovation represented by designing a system independent of the specific network architecture. In a subsequent phase, this can be achieved through a vehicular communication network or a specific protocol, such as the IEEE $802.11 \mathrm{p}$ standard. Moreover, the proposed solution allows creating an optimal design method that can be employed practically in any scenario.

Therefore, everything concerning the communication and reception of information will be represented as a "black box," which, starting from appropriate input signals, will provide specific commands at the output to control the intelligent traffic light system, as shown in Figure 2. The inputs provided to the black box may come from any part of the network infrastructure. At the same time, the output is the control commands of the intelligent traffic light. The system dynamically manages the various traffic flows developed in the separate roads, also taking into account specific priorities, with higher priority for emergency vehicles or vehicles with longer waiting times in queue. Having

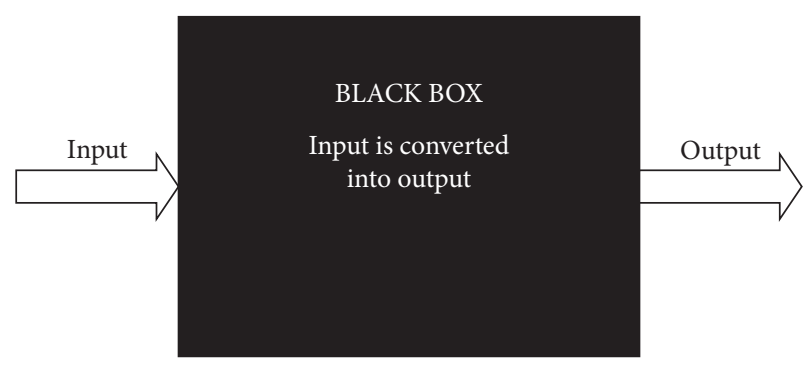

FIGURE 2: Schematic representation of the network infrastructure.

set the initial conditions the problem is to evaluate the vehicular traffic present in a four-way road intersection, through which different types of vehicles will pass.

The day is modeled as a random variable. Therefore, in the characterization of the variable relating to traffic flows, it could be helpful to start from a static condition, assuming that it always has identical functioning within a day. Subsequently, this variable is adapted to make it more consistent with the actual case. It is possible to define vehicular traffic from a statistical perspective, hence endowed with a probability density, a probability distribution, and all its moments. As a first approximation, it is reasonable to start from an ideal case in which the traffic peaks in specific time intervals of the day. Assuming the three peak times, respectively, at 08: $00 \mathrm{a}$ m., 01: $00 \mathrm{p} \mathrm{m}$., and 07: $30 \mathrm{p} \mathrm{m}$., it is possible to identify the probability density of traffic with a Gaussian function that assumes the following specific characteristics:

(i) A uniformly distributed probability distribution with constant amplitude throughout the night

(ii) Three different Gaussian probability distributions for peak hours

(iii) A uniform probability distribution with constant amplitude over the rest of the day (even of a different value than the first case)

The Gaussian distribution is uniquely identified by two parameters: the mean value $(\mu)$ and the standard deviation $(\sigma)$. The average value $\mu$, in the case considered in this work, is represented by the peak time value, which is equivalent to the central value of the distribution itself. The curve will be symmetrical concerning this value $[22,23]$. As the average value diversifies, a horizontal translation of the symmetry axis of the curve itself is obtained (case (a) in Figure 3). The standard deviation $\sigma$ represents the "peak" of the curve: the more significant the deviation, the greater the width of the peak. Therefore, the resulting graph will be more flattened. As the value of the standard deviation decreases, the curve will be more pointed (although it will be narrower in time, as in the case (b) in Figure 3).

Therefore, it is possible to state that the lower the variance value, the shorter the duration of the curve of the same Gaussian distribution. In this way, the peak hours will be temporally reduced, with a consequent increase in the maximum peak. Conversely, the greater the standard deviation value, the greater the temporal duration of vehicular 


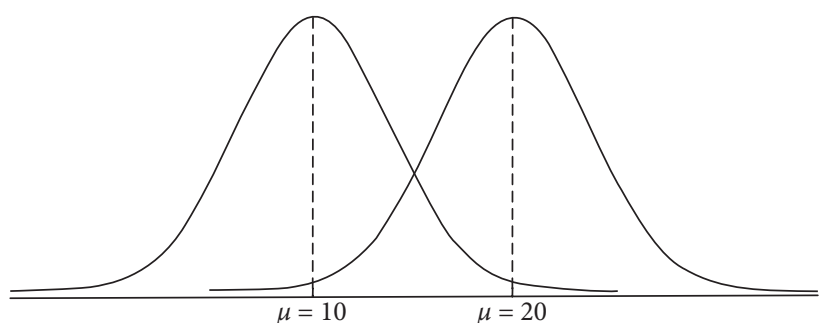

(a)

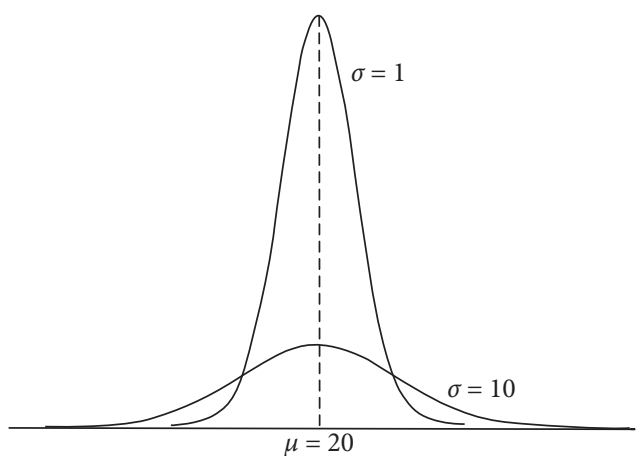

(b)

Figure 3: Changes of a Gaussian distribution as $\mu$ and $\sigma$ vary.

traffic, even if it will be less spiked in terms of the maximum value (case (b) in Figure 3 as $\sigma$ varies) [16].

In this work's hypothesis, the relative mean value and standard deviation values have been appropriately set and then analyzed using Matlab. Assuming that the peak times of maximum vehicular traffic occur strictly at $08: 00$ a m. (Case 1), at $01: 00 \mathrm{p} \mathrm{m}$. (Case 2), and, finally, at $07: 30$ $\mathrm{p} \mathrm{m}$. (Case 3), it will be necessary to set three different values of $\mu$ (in particular, it will be $\mu 1=28,800 s, \mu 2=$ $46,800 \mathrm{~s}$, and $\mu 3=70,200 \mathrm{~s}$ ). As far as the value of $\sigma$ is concerned, it is permanently fixed at the same value in all three cases, i.e., equal to $1,800 \mathrm{~s}$ (i e., 30 minutes). This situation means that the three peaks representing the different peak hours will have a total duration of one, precisely:

(i) In case 1, the peak hour will start at 07:30 a.m. and finish at $08: 30$ a.m.

(ii) In case 2, the peak hour will start at 12:30 p.m. and finish at $01: 30$ p.m.

(iii) In case 3, the peak hour will start at 07:00 p.m. and finish at 08:00 p.m.

Under these hypotheses, the random variable's probability density that simulates the vehicular traffic present in each of the experimental cases in question illustrated in shown in Figure 4. It is possible to establish variability during the day of the traffic flow. In particular, it will assume peak values in specific time intervals due to the greater concentration of vehicles along the roads. This circumstance is easily explained by specific habits that regulate people's lives (time of commuting to and from work, time of starting the children's school, lunch break, leaving school for children).

\section{Performance Evaluation}

The comparison between the three construction hypotheses (reported in subsection C) is based on the average times and maximum waiting times in the queue of a generic vehicle located along a specific carriageway. In particular, the implementation of each of the three hypotheses is along the following three scenarios: (1) the scenario is a static traffic light system, in which, therefore, the green times and their duration will be

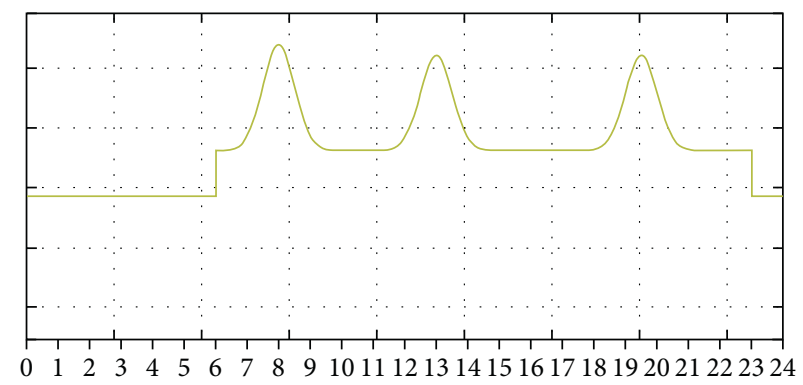

Figure 4: Probability density of the traffic variable during day.

preset, regardless of the actual traffic conditions; (2) the second scenario, this time fully implemented, in which the network infrastructure uses the IEEE 802.11p; finally, (3) a third scenario, consistently fully implemented, will use vehicular communications (LTE-V2V) as network infrastructure (this application is perfectly compatible with communications in 5G cellular networks).

Before comparing the average times and maximum average waiting times in the queue, a brief comparison is also made below between the networks that use the IEEE $802.11 \mathrm{p}$ protocol and LTE-V2V vehicular communications in terms of performance parameters [17]. The comparison of different standards, valid to support the applications of this work, is done in terms of transmission speed, signal coverage, signal interference, accessibility, maintenance, initial costs, and safety [18-20].

First of all, it can be observed that both standards analyzed operate on frequencies around $5.9 \mathrm{GHz}$ (working frequency band). It can also be observed that wireless standards that operate on such high-frequency bands are less affected by interference and absorption (due to atmospheric phenomena such as water and fog). Some protocols are based on the contention of the transmission medium, thus making it possible to establish possible collisions. However, these standards allow quick access to the requested service. In general, wireless standards that support long-range transmissions have higher initial costs than standards that support shortrange communications; moreover, the latter lead to lower maintenance costs [21]. 
Although some wireless standards use particular encryption and authentication mechanisms to guarantee the transmission of secure communications, they are still vulnerable to any external security breaches (hackers). However, most standards provide highly secure data transmissions [22, 23].

Considering the analysis carried out in [24], the two different technologies are compared under identical external conditions, that is, in an urban setting. From the experimental results achieved, it is possible to recognize that LTE-V2V can provide a $10 \%$ improvement in the packet reception ratio and a ten times lower update delay than IEEE 802.11p. However, these results are obtained only in conditions of low traffic data. The situation changes when a more stressful traffic condition, in terms of packets sent and received, is considered. Under these conditions, while the LTE-V2V works better regarding the packet reception ratio (better than 26\%), the IEEE 802.11 p guarantees a lower update delay.

For comparison, it is also helpful to estimate the results of using different modulation and coding schemes (MCS). The different strategies used can significantly impact the quality of service (QoS) and the coverage area. In [25-27], it was established that the use of IEEE 802.11p allows for exceptional performance up to a few hundred meters, intended as a coverage range. It is reasonable to think that a vehicle can be identified every 10 meters and that, therefore, in a range of 250-300 meters, around 30 vehicles can be identified. Due to the excessive collision speed, the reliability of the communication tends to undergo a substantial decrease, especially in applications with distances more significant than those just mentioned (a few hundred meters) [28]. All other things being equal, it can be verified that the LTE-V2V standard has reduced performance in short distance communications but is more reliable as the distance increases (for example, even up to almost 500 meters) $[29,30]$. In light of this, it is not easy to establish which of the two standards results in better performance $[31,32]$. Therefore, depending on the conditions of use and the precise characteristics required by the application to be developed, it is possible to choose one of the two standards [24].

4.1. Traffic Generator. In the Matlab/Simulink simulation environment using TrueTime [33], to generate the variable relating to the vehicular traffic existing in a given road (this is a random variable characterized by a probability density that varies over time), a real number generator was used to generate on the interval [0-0.7]. The characteristic of this sequence of numbers depends on the initial seed: when this initial seed varies, a completely different numerical sequence follows in which there is no mathematical relationship between one number and another; instead, if the seed is kept from one simulation to another, the generated sequence will be the same with consequent generation of the same traffic configuration [34]. Since, in the case under examination, the generation of eight tails, there are eight generators of pseudorandom numbers with different seeds (values between 1 and 8).

It is necessary to consider a waveform that examines the traffic probability density, which, together with the number generator described above, follows an IF-THEN construct. If at instant $t$ their sum exceeds a certain threshold (set at 0.79 ), then a vehicle will be generated at the same instant, otherwise, no. At the instant $(t+1)$, the same check is performed, and so on. It is much more likely that their sum will exceed the threshold value at peak times, while, at night, it is less likely that this threshold will be exceeded. The system described is represented in Figure 5.

The input variables of the TrueTime Kernel [33], except for the Uniform Random Number, are constants that shape the waveform of the traffic probability density. When one of them varies, it is possible to operate on efficiently:

(1) The probability that a vehicle will arrive at the intersection between $11: 00 \mathrm{pm}$ and $00: 00$ and between 00:00 and 06:00 am (constant night)

(2) The probability that a vehicle will arrive at the intersection between 06:00 am and 11:00 pm (constant morning)

(3) The probability that a vehicle will arrive at the intersection during the peak hours at 08:00 am (Gaussian multiplicative constant 1)

(4) The probability that a vehicle will arrive at the intersection during the peak hours $01.00 \mathrm{pm}$ (Gaussian multiplicative constant 2)

(5) The probability that a vehicle will arrive at the intersection during the peak hours of $7.30 \mathrm{pm}$ (Gaussian multiplicative constant 3)

(6) The duration of the peak hours at 8am (Gaussian variance 1 )

(7) The duration of the peak hours at 01:00 pm (Gaussian variance 2)

(8) The duration of the peak hours at $07: 30 \mathrm{pm}$ (Gaussian variance 3)

After setting these parameters and the initial seed of the random number generator [35], the TrueTime Kernel executes the following instructions every second:

(i) Determines the value of the probability density at instant $t$

(ii) Acquires the number generated randomly from input 1

(iii) If their sum is more significant than 0.79 , send a vehicle out; otherwise, wait for the next instant

The second TrueTime Kernel has the objective of performing a simple subtraction between the generated vehicles and the disposed vehicles (counted in the other TrueTime Kernel and forwarded to the one understudy). Thus, it is possible to know moment by moment the length of queues subsequently displayed. 


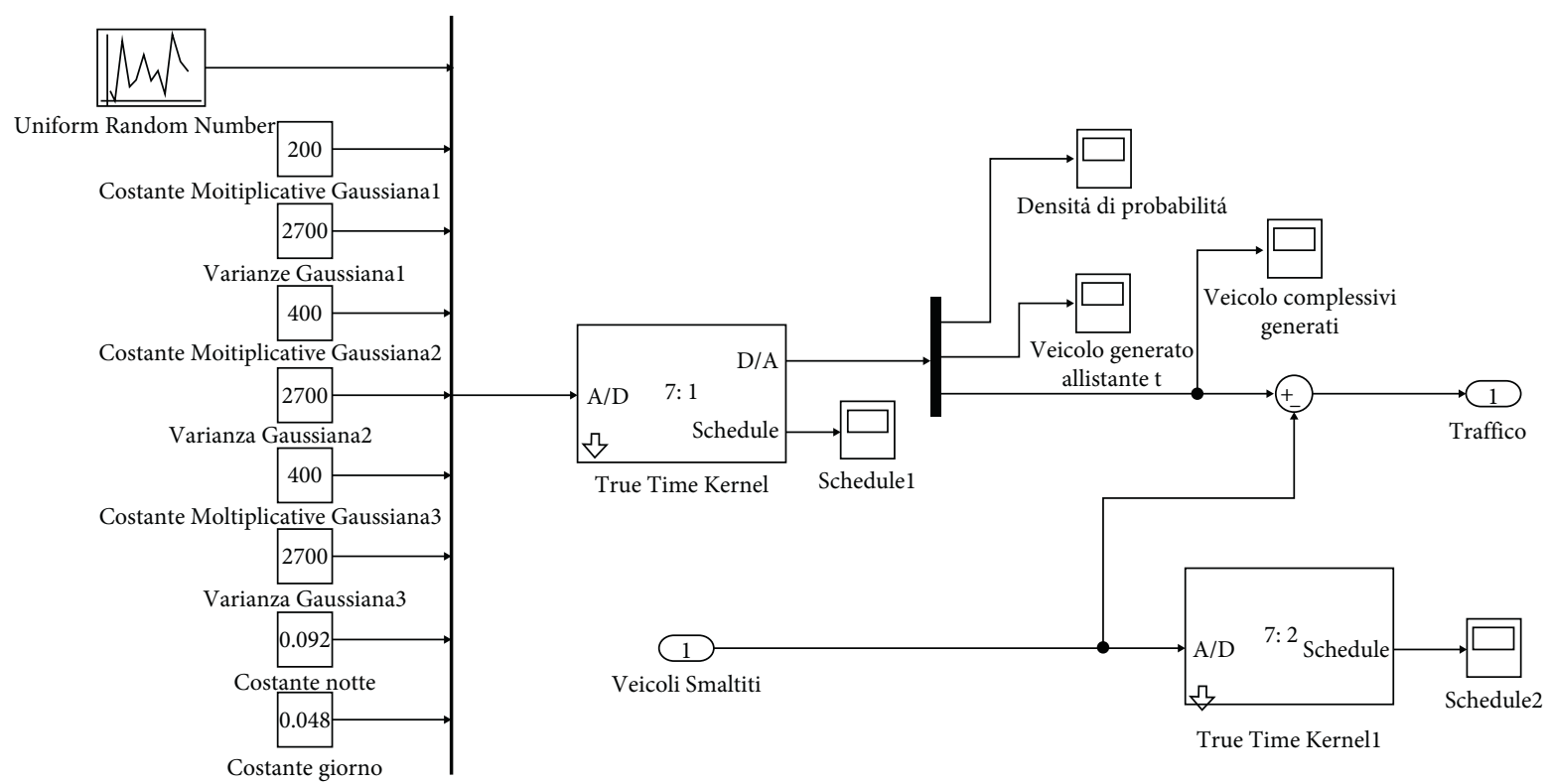

Figure 5: Schematic of the traffic generator in the Matlab/Simulink and TrueTime environment.

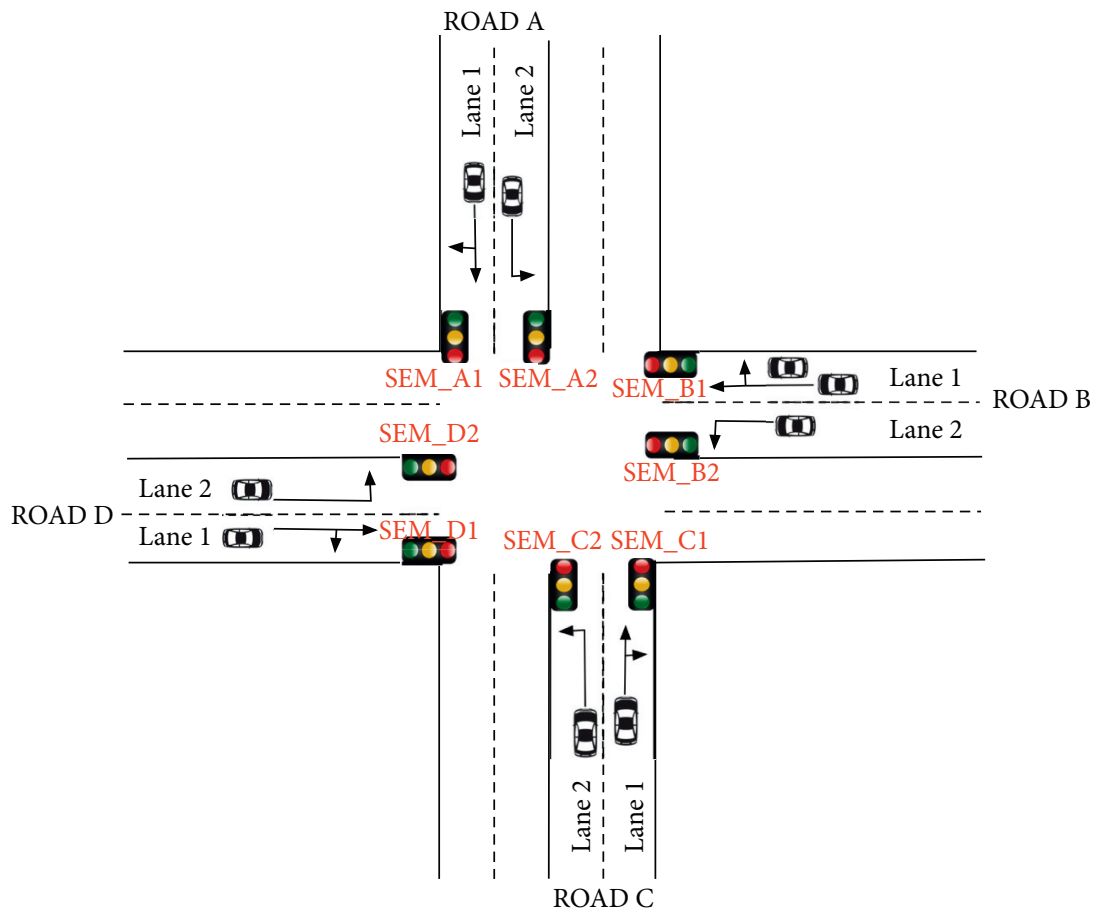

FIgURE 6: The road intersection object of this case study.

4.2. Evaluation Scenarios. The solution proposed in this work has been implemented to be independent of the input signals (i.e., from the network infrastructure) and to allow for the output of signals equally independent from the actuators that will act on the intelligent traffic lights. Therefore, a system that is not affected by the technology can be considered independent for any application. Furthermore, as shown in Figure 6, the traffic lights are managed in such a way as to prevent vehicles coming from different lanes of the same road from accessing the intersection simultaneously. This choice has been made to avoid specific points of conflict that could occur when the vehicle trajectories from the two affected lanes intersect. In this way, as shown in Figure 6, the cars coming from the two lanes of road A cannot go straight and turn left, respectively, during the same phase. 

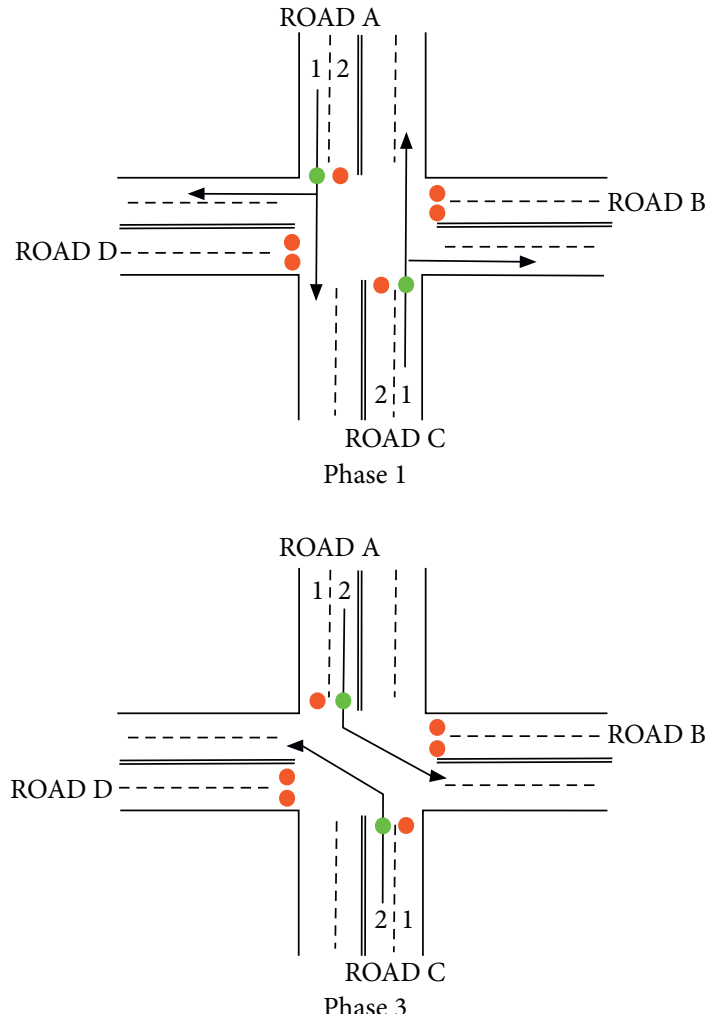
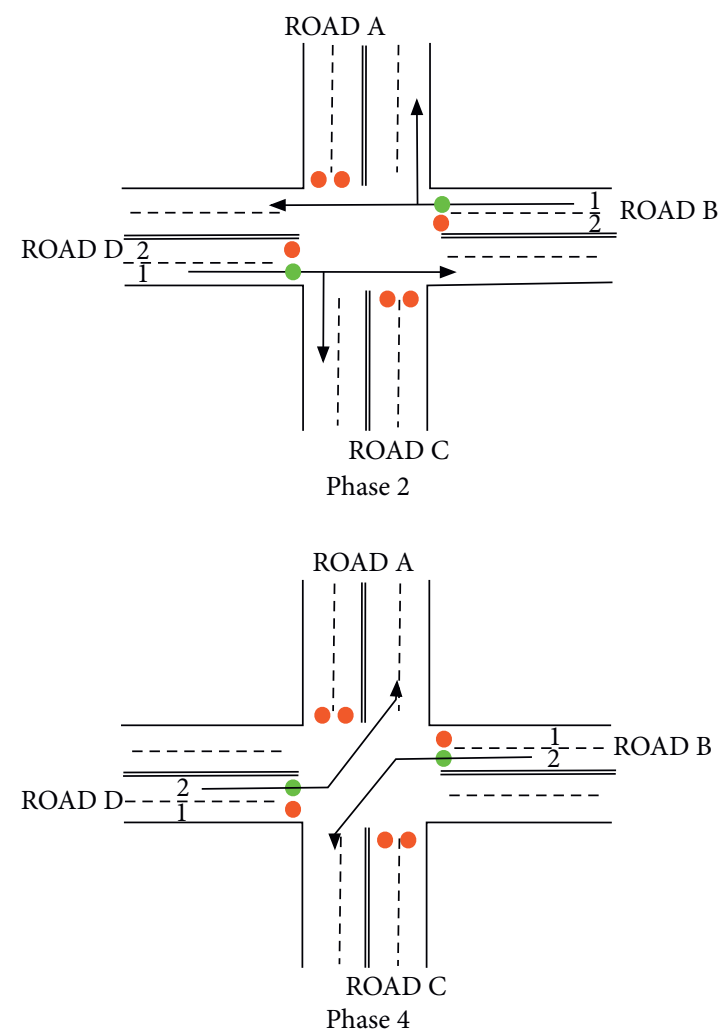

Figure 7: Traffic light phases.

The intersection is characterized by four phases, as illustrated in Figure 7.

To complete the configuration, whose performances will be evaluated later, it is necessary to note once again that a traffic light phase represents the period during which a specific set of lanes can continue following the proper direction. In this work, the following has been assumed:

(1) when phase 1 is enabled, only the cars of lane 1 of roads $\mathrm{A}$ and $\mathrm{C}$ can go straight or turn right, while all the other lanes will have a red light

(2) when phase 3 is enabled, only the cars of lane 2 of roads $\mathrm{A}$ and $\mathrm{C}$ can turn left, while all the other lanes will have a red light

(3) when phase 2 is enabled, only the cars of lane 1 of roads $B$ and $D$ can go straight or turn right, while all the other lanes will have a red light

(4) when phase 4 is enabled, only the cars of lane 2 of roads $B$ and $D$ can turn left, while all other lanes will have a red light

4.3. Obtained Results. Before acquiring and comparing the data relating to the average and maximum overall waiting times of each simulation, it is necessary to define what the waiting time of a vehicle represents and which parameters influence it. Specifically, the waiting time for a vehicle is the difference between the vehicle's instant arrival and how the vehicle clears a specific intersection $[36,37]$.

To know the instant when a vehicle occurs in an intersection, the following arrays were used (an array can be imagined as a container of elements of the same type, having a variable-length; in the case in question, the elements the array is composed of will be described in the following):

(i) An array A in which, every second, in the i-th position 1 is entered if in the i-th instant a vehicle is presented or 0 otherwise.

(ii) An array B that varies its size every second by adding an element constructed as follows: if at the $i$-th instant a vehicle arrives, or when the outbound traffic generator sends the value 1 , then, in the i-th position of the array $\mathrm{B}$, the value of the current instant will be written using the ttCurrentTime function. On the contrary, if the outbound traffic generator sends the value 0 , the value 0 is inserted for convenience in the $\mathrm{i}$-th position of array $\mathrm{B}$.

(iii) An array $\mathrm{C}$ containing the time values in which each vehicle clears the intersection. To construct this number vector, it is necessary to proceed according to the following procedure: first of all, it is necessary to 

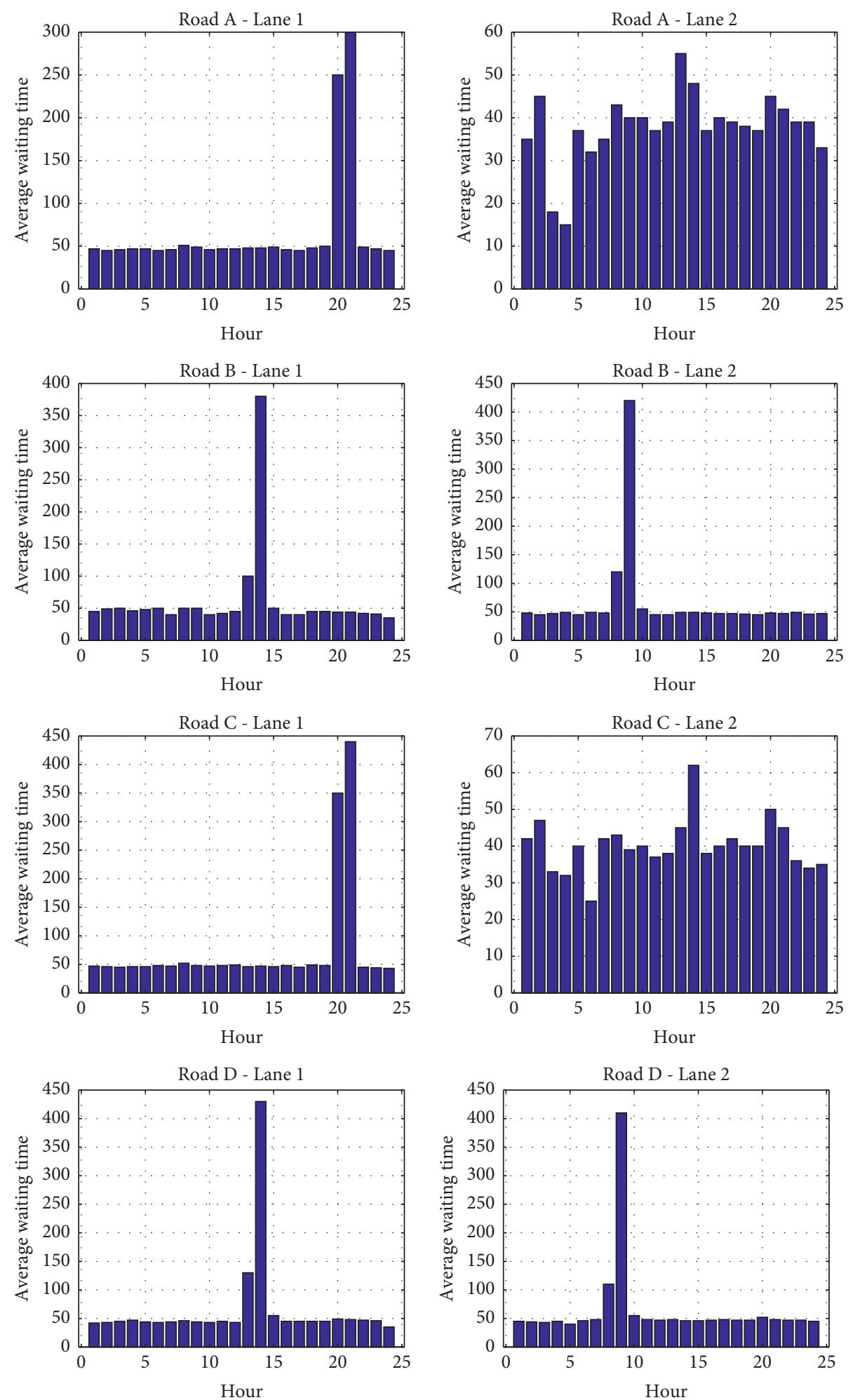

FIgURE 8: Average waiting times in the queue (case 1). 
TABLE 1: Average waiting times of vehicles in the approach with static traffic lights.

\begin{tabular}{|c|c|c|c|c|c|c|c|c|}
\hline & A1 & A2 & B1 & B2 & $\mathrm{C} 1$ & $\mathrm{C} 2$ & D1 & D2 \\
\hline Hour 1 & 41.5741 & 33.4001 & 33.7144 & 38.3543 & 38.2487 & 42.001 & 32.2728 & 35.9774 \\
\hline Hour 2 & 36.5633 & 44.5456 & 45.1112 & 32.8637 & 39.7701 & 47.6668 & 39.0001 & 34.7956 \\
\hline Hour 3 & 40.354 & 19.2001 & 47.8126 & 36.7074 & 38.2807 & 33.143 & 32.6001 & 33.8538 \\
\hline Hour 4 & 41.0279 & 17.889 & 37.5715 & 40.5919 & 38.3141 & 32.8334 & 34.1861 & 35.2746 \\
\hline Hour 5 & 40.8855 & 37.6668 & 40.5001 & 33.2808 & 38.2021 & 40.4287 & 30.9788 & 29.3334 \\
\hline Hour 6 & 39.4311 & 31.8001 & 20.6251 & 41.1065 & 38.1041 & 25.7274 & 36.4232 & 37.5218 \\
\hline Hour 7 & 39.7637 & 37.2842 & 38.5724 & 40.8036 & 36.3207 & 42.0738 & 40.5388 & 40.8399 \\
\hline Hour 8 & 52.8131 & 43.17 & 55.3553 & 111.1956 & 51.8183 & 42.5682 & 45.6659 & 108.0836 \\
\hline Hour 9 & 44.9469 & 41.3694 & 55.9848 & 402.7664 & 44.7912 & 39.873 & 44.2679 & 406.9663 \\
\hline Hour 10 & 38.2421 & 41.5319 & 39.5875 & 58.6872 & 40.418 & 39.9615 & 40.2076 & 71.2615 \\
\hline Hour 11 & 39.2453 & 39.0726 & 40.0238 & 41.3272 & 43.8832 & 38.8179 & 37.4836 & 39.4614 \\
\hline Hour 12 & 39.2095 & 40.4651 & 42.9501 & 41.0429 & 38.8634 & 39.8243 & 43.8407 & 39.3631 \\
\hline Hour 13 & 41.3113 & 56.0615 & 96.8351 & 46.7232 & 41.0408 & 47.8793 & 126.139 & 41.9496 \\
\hline Hour 14 & 41.9149 & 48.8504 & 385.161 & 46.143 & 38.9263 & 60.4365 & 423.2484 & 42.8778 \\
\hline Hour 15 & 40.0846 & 39.8517 & 59.3673 & 41.5339 & 40.3553 & 38.5032 & 71.2244 & 40.6001 \\
\hline Hour 16 & 40.2519 & 41.762 & 37.2796 & 40.1788 & 40.1357 & 41.1993 & 40.0803 & 38.9742 \\
\hline Hour 17 & 36.7173 & 41.5315 & 38.1823 & 40.635 & 40.8862 & 41.8294 & 39.9586 & 39.8123 \\
\hline Hour 18 & 41.2501 & 41.0967 & 43.26 & 39.3714 & 44.6591 & 40.9616 & 39.5416 & 40.0431 \\
\hline Hour 19 & 46.6958 & 44.222 & 42.933 & 43.0649 & 45.04 & 41.0365 & 39.3824 & 45.3754 \\
\hline Hour 20 & 257.6708 & 48.7209 & 42.4555 & 41.2396 & 387.5844 & 50.0956 & 51.09 & 57.0642 \\
\hline Hour 21 & 300.8803 & 43.3334 & 42.9705 & 45.1953 & 446.1559 & 45.5201 & 46.1992 & 43.0261 \\
\hline Hour 22 & 42.1793 & 40.3746 & 40.1515 & 39.1984 & 40.2942 & 38.9121 & 40.3647 & 41.2483 \\
\hline Hour 23 & 41.2359 & 40.2442 & 38.8771 & 39.6965 & 37.7105 & 37.8296 & 41.3347 & 39.9879 \\
\hline Hour 24 & 38.4386 & 31.4168 & 29.3334 & 37.353 & 35.2279 & 38.2728 & 25.8751 & 36.8294 \\
\hline
\end{tabular}

check whether at the i-th instant a vehicle has cleared the intersection: if so, using a FOR construct, one searches for the first 1 present in the array $\mathrm{A}$, set to 0 , and insert the current time in $\mathrm{C}$, that is, the instant in which this operation was performed and finally insert the value in the i-th position of array $\mathrm{C}$; otherwise, always in the $\mathrm{i}$-th position of array $\mathrm{C}$, the value 0 is entered. Array A is used to make this calculation accurate since the First Come First Served (FCFS) policy applies at road intersections.

(iv) An array $D$ was obtained at the end of the simulation, as the difference between array $\mathrm{C}$ and array $\mathrm{B}$. D will contain the waiting times of each vehicle and several zeros, which will not affect the average calculation.

To perform the average of the waiting times hour by hour, in order to display the histograms, a counter $n_{h}$ that counts the nonzero waiting times in array $D$ is used. The calculation is performed in stock of 3600 items (i.e., in one hour). For fixed $n_{h}$, the $Y$ array of average values is obtained as shown in

$$
Y[h]=\frac{1}{n_{h}} \sum_{i=0}^{3600} D[i+3600 h],
$$

where $h \in \backslash\{0,1,2, \ldots, 23 \backslash\}$ and takes into account the time in which the average waiting time is being calculated. To give an idea, to calculate the average waiting time in the hour between $00: 00: 00$ and $01: 00: 00$, where $h$ is $0, Y[1]$ is shown in

$$
Y[1]=\frac{1}{n_{1}} \sum_{i=0}^{3600} D[i],
$$

in which the first 3600 seconds of waiting is considered. Subsequently, once the first hour has ended, and the next one has begun, which goes from $01: 00: 00$ to $02: 00: 00, h$ is increased by 1 , and the sum becomes as shown in

$$
Y[2]=\frac{1}{n_{2}} \sum_{i=0}^{3600} D[i+3600],
$$

in which the waiting times of individual vehicles that show up between 01:00:00 and 02:00:00 are considered.

Once the calculation of the 24 average waiting times in 24 hours $Y$ [1:24] is completed, this is given as input value to the Matlab $\operatorname{bar}(X, Y)$ function, where $X=[1: 24] \mathrm{fa}$. This function draws columns in the histogram with a height equal to the value of $Y[X]$ in the positions indicated by the array $X$.

The values $Y[X]$ are saved to a file, and the histograms are stored as images. The construction of the histograms above was repeated for all eight lanes present in the case study in question. It also seems appropriate to specify that the various simulations were consistently implemented with TrueTime. The different networks were developed using the TrueTime Wireless Network, customized and adapted from time to time based on the chosen network architecture.

Figure 8 shows the histograms relating to the average waiting times of the intersection equipped with static traffic lights (Case 1). In this scenario, the average waiting time is 56.23 seconds. 

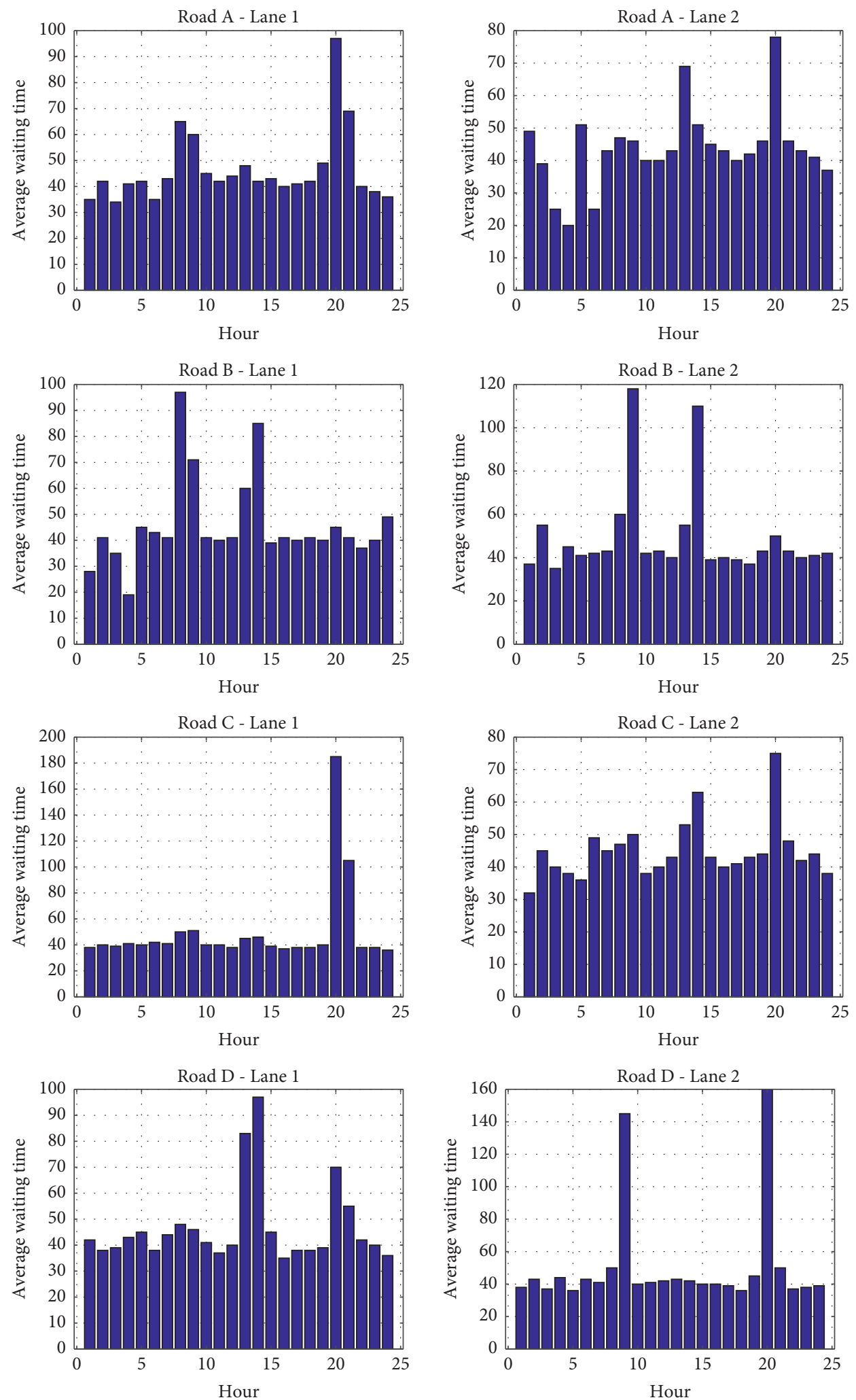

FIgURE 9: Average waiting time in the queue (Case 2). 
TABLE 2: Average waiting time of vehicles in the approach with standard IEEE 802.11p.

\begin{tabular}{|c|c|c|c|c|c|c|c|c|}
\hline & A1 & A2 & B1 & B2 & $\mathrm{C} 1$ & $\mathrm{C} 2$ & D1 & D2 \\
\hline Hour 1 & 35.9764 & 48.6001 & 29.0001 & 35.2293 & 35.712 & 32.9334 & 42.7046 & 37.6592 \\
\hline Hour 2 & 42.3317 & 38.091 & 40.5557 & 50.7956 & 39.0101 & 45.6668 & 37.2668 & 46.6137 \\
\hline Hour 3 & 35.9215 & 26.4001 & 35.8126 & 33.1464 & 38.2654 & 41.2144 & 38.9001 & 35.4879 \\
\hline Hour 4 & 41.1334 & 21.6668 & 21.2858 & 48.143 & 42.8199 & 39.1668 & 44.1861 & 49.6668 \\
\hline Hour 5 & 42.8022 & 51.0668 & 46.8572 & 43.3685 & 41.5153 & 38.0715 & 47.1278 & 34.6276 \\
\hline Hour 6 & 36.4484 & 28.4001 & 44.6251 & 44.6171 & 45.5781 & 49.3637 & 38.3463 & 47.4566 \\
\hline Hour 7 & 43.8851 & 43.2215 & 43.0708 & 44.7538 & 43.1708 & 45.7089 & 45.0775 & 43.7212 \\
\hline Hour 8 & 65.433 & 48.0865 & 97.5676 & 61.5833 & 55.3993 & 47.7724 & 48.9746 & 68.6195 \\
\hline Hour 9 & 62.5625 & 47.6194 & 74.1051 & 115.6145 & 56.0296 & 50.3617 & 47.1944 & 144.4308 \\
\hline Hour 10 & 47.1852 & 40.8503 & 41.8217 & 45.2578 & 42.5822 & 40.9113 & 42.3164 & 42.7075 \\
\hline Hour 11 & 45.1265 & 40.9505 & 40.0792 & 45.8737 & 42.5566 & 42.9747 & 38.4836 & 43.3876 \\
\hline Hour 12 & 46.7041 & 43.8027 & 42.556 & 44.7035 & 40.6008 & 45.9698 & 42.6407 & 44.7823 \\
\hline Hour 13 & 50.3955 & 69.4833 & 61.256 & 58.0221 & 48.5611 & 54.1526 & 83.4903 & 45.5772 \\
\hline Hour 14 & 45.3655 & 51.0417 & 88.3152 & 108.79 & 49.0384 & 64.3658 & 97.2875 & 44.79 \\
\hline Hour 15 & 46.4438 & 45.1722 & 40.2508 & 41.3474 & 41.7684 & 45.0277 & 46.9476 & 43.3475 \\
\hline Hour 16 & 41.6943 & 44.4326 & 44.2206 & 42.0171 & 39.0382 & 42.4473 & 37.1261 & 43.3234 \\
\hline Hour 17 & 42.2419 & 40.9415 & 42.5224 & 41.552 & 42.0072 & 43.1424 & 41.2454 & 42.7144 \\
\hline Hour 18 & 43.0834 & 43.6553 & 44.1987 & 39.1288 & 42.1542 & 45.5424 & 41.3598 & 39.6273 \\
\hline Hour 19 & 50.0825 & 45.5289 & 42.6966 & 43.7378 & 45.9053 & 45.73 & 43.1912 & 47.2696 \\
\hline Hour 20 & 97.6618 & 78.0097 & 48.0892 & 49.4816 & 185.7501 & 75.7979 & 74.0844 & 159.6485 \\
\hline Hour 21 & 71.9042 & 47.6292 & 43.8758 & 43.883 & 111.6457 & 50.4701 & 57.5973 & 55.8495 \\
\hline Hour 22 & 42.2438 & 45.1012 & 39.2511 & 39.2322 & 40.7391 & 43.6041 & 44.3508 & 38.3483 \\
\hline Hour 23 & 40.3497 & 42.0513 & 43.8652 & 40.1661 & 40.5329 & 45.0776 & 42.464 & 38.9066 \\
\hline Hour 24 & 38.2607 & 32.8334 & 52.4445 & 40.4903 & 38.3408 & 41.0001 & 38.0501 & 39.5123 \\
\hline
\end{tabular}

By performing the arithmetic average between all the values of the widths of the columns of the histograms, the values included in Table 1 are obtained. It should be noted that, at peak times, the approach with a static traffic light system is unsuitable since very high waiting peaks can be encountered, even exceeding 400 seconds (more than 6 minutes).

The exact estimates were made with traffic conditions identical to the previous ones, applying the IEEE 802.11p standard as network infrastructure. Figure 9 displays the graphs relating to the average waiting times in this specific scenario (Case 2). By carefully analyzing the histograms, it can be immediately noticed that the waiting peaks of 400 seconds are eliminated; the highest is equal to 185.75 seconds, i.e., about 3 minutes. Furthermore, by calculating the average waiting time of all vehicles, it can be seen that it is lowered to 48.82 seconds, or 8 seconds less than in Case 1. The values of the individual average amplitudes of the histograms are depicted in Table 2.

In the third and last scenario, with a network implementation that uses LTE-V2V vehicular communications, the obtained average waiting times are presented in Figure 10 (Case 3 ). The overall average of the waiting time in the queue is, in this case, equal to 53.81 seconds, obtained by performing the arithmetic average between the values shown in Table 3. Although the average waiting time in the queue is slightly higher than in Case 2, it nevertheless represents a reasonable and competitive value. It should also be noted that this approach could exploit the existing (at least in a part of the national territory) 5G infrastructural network, thus providing for a lower cost in terms of the initial investment.

Therefore, from a careful analysis of the results obtained from the simulations, it is possible to establish that the approach relating to Case 1 has several disadvantages concerning average waiting times in the queue (there are peaks of 400 seconds, i.e., more than 6 minutes of waiting in the queue; moreover, the average waiting time is around one minute). Accordingly, this application can be considered the worst-case and empirically highlights how this approach is distant from the other two approaches, which, on the contrary, provide configurations in which intelligent traffic lights are exploited [38]. The analysis of Case 2, on the other hand, represents the best case, at least in terms of average waiting time in queues for a vehicle. In fact, in this scenario, the peaks present in the configuration of Case 1 are eliminated so much, so that the maximum peak present is around 3 minutes (worst-case equal to 185 seconds), while the average waiting time in the queue drops drastically by almost 10 seconds, reaching an average time of about 49 seconds. The third approach (Case 3) represents the intermediate case as it allows for maximum peaks that reach about 200 seconds (i.e., about three and a half minutes). In comparison, the average waiting time in the queue 

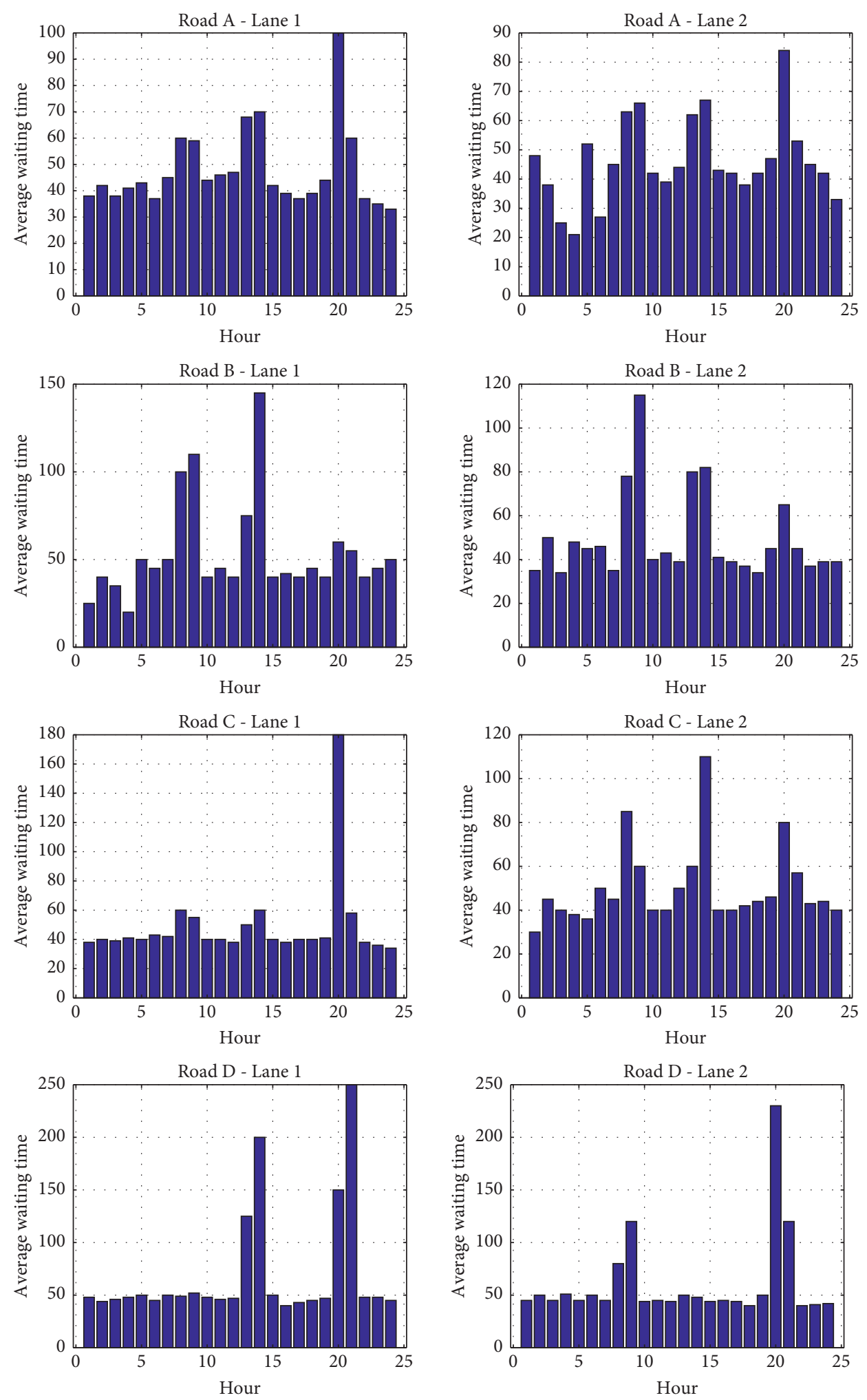

Figure 10: Average waiting time in the queue (Case 3). 
TABLE 3: Average waiting time of vehicles in the approach with LTE-V2V communications.

\begin{tabular}{|c|c|c|c|c|c|c|c|c|}
\hline & A1 & $\mathrm{A} 2$ & B1 & B2 & $\mathrm{C} 1$ & $\mathrm{C} 2$ & D1 & D2 \\
\hline Hour 1 & 35.9764 & 48.6001 & 29.0001 & 35.2293 & 35.712 & 32.9334 & 42.7046 & 37.6592 \\
\hline Hour 2 & 41.7948 & 38.091 & 40.5557 & 50.8865 & 39.0101 & 45.6668 & 37.3334 & 46.6365 \\
\hline Hour 3 & 35.9215 & 26.4001 & 35.8126 & 33.1464 & 38.2654 & 41.2144 & 38.9001 & 35.4879 \\
\hline Hour 4 & 41.1334 & 21.6668 & 21.5001 & 48.143 & 42.8199 & 39.1668 & 44.3257 & 49.6668 \\
\hline Hour 5 & 42.8022 & 51.0668 & 46.8572 & 43.4212 & 41.5153 & 38.0715 & 47.2554 & 34.6864 \\
\hline Hour 6 & 36.4484 & 28.4001 & 44.6251 & 44.6171 & 45.5781 & 49.3637 & 38.3463 & 47.4566 \\
\hline Hour 7 & 47.2844 & 44.4614 & 46.9261 & 35.5602 & 44.3555 & 46.1334 & 49.1584 & 35.6173 \\
\hline Hour 8 & 60.3314 & 62.1477 & 101.2065 & 74.9212 & 61.7728 & 87.364 & 47.9185 & 90.3741 \\
\hline Hour 9 & 59.9981 & 66.4092 & 106.197 & 112.324 & 56.5128 & 59.582 & 51.974 & 119.2214 \\
\hline Hour 10 & 46.235 & 42.0376 & 44.7176 & 43.8682 & 44.0538 & 42.309 & 43.5545 & 41.2444 \\
\hline Hour 11 & 49.479 & 38.1566 & 43.1226 & 45.6023 & 44.0324 & 41.8221 & 40.2895 & 43.4872 \\
\hline Hour 12 & 50.3719 & 46.223 & 46.7177 & 41.1805 & 41.7951 & 49.6546 & 42.1363 & 41.6734 \\
\hline Hour 13 & 68.2705 & 63.0041 & 74.1786 & 82.3254 & 55.1817 & 61.9874 & 130.3677 & 53.9762 \\
\hline Hour 14 & 70.0331 & 67.0957 & 144.0963 & 83.5085 & 64.481 & 107.9516 & 203.4032 & 48.4496 \\
\hline Hour 15 & 46.4895 & 44.19 & 42.8389 & 42.8779 & 42.6565 & 41.7056 & 50.4223 & 43.3159 \\
\hline Hour 16 & 43.3562 & 43.6826 & 43.3702 & 42.332 & 39.0382 & 41.4025 & 36.9238 & 43.7329 \\
\hline Hour 17 & 42.2788 & 39.9624 & 43.1297 & 41.2366 & 42.3738 & 42.5936 & 41.6001 & 42.3103 \\
\hline Hour 18 & 43.5452 & 44.8967 & 45.3972 & 38.3677 & 42.6755 & 45.227 & 42.3282 & 38.7062 \\
\hline Hour 19 & 49.5104 & 50.9754 & 43.3228 & 47.1513 & 46.3941 & 50.6417 & 46.3353 & 51.6248 \\
\hline Hour 20 & 101.2001 & 81.8586 & 66.7897 & 67.0371 & 183.1469 & 85.3728 & 147.105 & 237.3261 \\
\hline Hour 21 & 64.5774 & 54.6417 & 58.08 & 48.1503 & 62.9843 & 60.2076 & 253.5001 & 104.9326 \\
\hline Hour 22 & 40.8603 & 45.1874 & 40.9882 & 39.8398 & 41.1251 & 44.8761 & 44.1911 & 38.8149 \\
\hline Hour 23 & 40.3416 & 42.7639 & 44.2144 & 40.4495 & 39.865 & 45.3761 & 43.3613 & 39.1749 \\
\hline Hour 24 & 38.0636 & 33.0834 & 52.5557 & 40.4903 & 37.8188 & 41.091 & 38.2751 & 39.5123 \\
\hline
\end{tabular}

is around 54 seconds. However, the latter application allows integrating LTE technology with fifth-generation (5G) cellular communications. As has already been mentioned, this could represent a significant advantage for this particular approach. It would provide the possibility of using the $5 \mathrm{G}$ infrastructures (partly already existing) instead of the case involving the use of the 802.11 p standard.

\section{Conclusions}

This work presented an innovative approach to the managing an intelligent traffic light system. The central basis of this work is the implementation of an intelligent traffic light whose management is required from three application scenarios. The decision-making part was set as a black box inside which input signals enter, and output signals are produced. All this made it possible to imagine three different realization hypotheses, simulated through Matlab, and provided valuable data for their subsequent comparison.

The proposed approach has brought out a series of advantages and innovations compared to the multiple solutions existing in the literature. First of all, there is the generality of the treatment making any implementation solution independent from the point of view of the network infrastructure that is implemented upstream. Another fundamental aspect is represented by the transparency of the functioning of the black box concerning the infrastructural solution. In this sense, this block does not affect the performance of the network used, regardless of what the block is. The simplicity of implementation is another essential advantage introduced by the approach reported in this work; a simple input-output block results in a considerable saving of energy and cost of the final solution adopted.
From a detailed analysis of the data, it seems possible to establish that the best implementation solution, from an infrastructural point of view, is that of the 802.11p standard. However, it is necessary to take into consideration the fact that the LTE-V2V technology, as already described in this work, could be a technologically more attractive solution, given that it is ready to be adapted to $5 \mathrm{G}$ technology, whose infrastructure is already, at least in part, widespread. This situation would make it possible to lower the initial economic impact significantly. However, it is also important to point out that the $802.11 \mathrm{p}$ standard is currently subject to continuous evolutions.

Therefore, based on this, this work it can be concluded that none of the multiple applications analyzed is universally best. On a case-by-case basis, it is necessary to choose which solution introduces more significant advantages over developing a particular application. The comparison with other implementation hypotheses, the possibility of making the services provided by an intelligent traffic light system useable also by autonomous vehicles (AV), and the ability to make new implementation approaches (also in terms of developing new standards of communication) are all possible topics that could be developed as further study of this research topic.

\section{Data Availability}

The data are not made available since the research has been incorporated into a research project that involves the presentation of patents.

\section{Conflicts of Interest}

The authors declare that they have no conflicts of interest. 


\section{References}

[1] S. Kelathodi Kumaran, D. Prosad Dogra, and P. Pratim Roy, "Queuing theory guided intelligent traffic scheduling through video analysis using dirichlet process mixture model," Expert Systems with Applications, vol. 118, pp. 169-181, 2019.

[2] Asutos Inc, "American multi-nation Engineering \& Infrastructure company," 2022, www.asutos.com/.

[3] A. Kanungo, A. Sharma, and C. Singla, "Smart traffic lights switching and traffic density calculation using video processing," in Proceedings of the 2014 Recent Advances in Engineering and Computational Sciences (RAECS), pp. 1-6, Chandigarh India, March 2014.

[4] M. Collotta, L. Lo Bello, and G. Pau, "A novel approach for dynamic traffic lights management based on wireless sensor networks and multiple fuzzy logic controllers," Expert Systems with Applications, vol. 42, no. 13, pp. 5403-5415, 2015.

[5] S. Sharma, A. Pithora, G. Gupta, M. Goel, and M. Sinha, "Traffic light priority control for emergency vehicle using rfid," International Journal of Innovations in Engineering and Technology, vol. 2, no. 2, 2013.

[6] B. Zhou, J. Cao, X. Zeng, and H. Wu, "Adaptive traffic light control in wireless sensor network-based intelligent transportation system," in Proceedings of the 2010 IEEE 72nd Vehicular Technology Conference - Fall, pp. 1-5, Ottawa, ON, Canada, September, 2010.

[7] D. Sun, R. Jiang, and B.-H. Wang, "Timing of traffic lights and phase separation in two-dimensional traffic flow," Computer Physics Communications, vol. 181, no. 2, pp. 301-304, 2010.

[8] T. T. Ngo, T. Huynh-The, A. Abdukhakimov, and D. S. Kim, "A novel vanets-based traffic light scheduling scheme for greener planet and safer road intersections," IEEE Access, vol. 7, no. 1-1, 2019.

[9] L. Wu, L. Nie, S. U. Khan, O. Khalid, and D. Wu, “A v2i communication-based pipeline model for adaptive urban traffic light scheduling," Frontiers of Computer Science, vol. 13, no. 5, pp. 929-942, 2019.

[10] H. Garg and E. G. Kaushal, "Traffic lights control system for indian cities using wsn and fuzzy control," International Research Journal of Engineering and Technology (IRJET), vol. 4, no. 7, pp. 2587-2592, 2017.

[11] M. Friesen, R. Jacob, P. Grestoni, T. Mailey, M. R. Friesen, and R. D. McLeod, "Vehicular traffic monitoring using bluetooth scanning over a wireless sensor network," Canadian Journal of Electrical and Computer Engineering, vol. 37, no. 3, pp. 135-144, 2014.

[12] P. Keeratiwintakorn, "Ubiquitous communication for $\mathrm{v} 2 \mathrm{v}$ and v2i for Thailand intelligent transportation system," in Proceedings of the NTC International Conference, Orlando FL, USA, May 2021.

[13] Z. Niu, W. Yao, Q. Ni, and Y. Song, "Study on qos support in 802.11e-based multi-hop vehicular wireless ad hoc networks," in Proceedings of the IEEE International Conference On Networking, Sensing And Control, ICNSC 2007, pp. 705-710, IEEE, London UK, April 2007.

[14] H. T. Cheng, H. Shan, and W. Zhuang, "Infotainment and road safety service support in vehicular networking: from a communication perspective," Mechanical Systems and Signal Processing, vol. 25, no. 6, pp. 2020-2038, 2011.

[15] S. Trubia, A. Severino, S. Curto, F. Arena, and G. Pau, "On brt spread around the world: analysis of some particular cities," Infrastructure, vol. 5, no. 10, 2020.

[16] A. E. Gomez, F. A. R. Alencar, P. V. Prado, F. S. Osório, and D. F. Wolf, "Traffic lights detection and state estimation using hidden Markov models," in Proceedings of the 2014 IEEE Intelligent Vehicles Symposium Proceedings, pp. 750-755, Dearborn, MI, USA, June, 2014.

[17] T. Kayarga and T. M. Sushma, "A survey on vanet technologies," International Journal of Computer Application, vol. 121, no. 1-9, 2015.

[18] B. Bilgin and V. Gungor, "Performance comparison of ieee 802.11p and ieee 802.11 b for vehicle-to-vehicle communications in highway, rural, and urban areas," International Journal of Vehicular Technology, vol. 2013, Article ID 971684, 11 pages, 2013.

[19] G. Cecchini, A. Bazzi, B. M. Masini, and A. Zanella, "Performance comparison between ieee 802.11p and lte-v2v incoverage and out-of-coverage for cooperative awareness," in Proceedings of the 2017 IEEE Vehicular Networking Conference (VNC), pp. 109-114, Turin, Italy, November, 2017.

[20] S. Chen, W. Nai, D. Dong, W. Zheng, and W. Jing, "Key indices analysis of ieee $802.11 \mathrm{p}$ based vehicle to infrastructure system in highway environment," Procedia - Social and Behavioral Sciences, vol. 96, pp. 188-195, 2013.

[21] F. Arena and D. Ticali, "The development of autonomous driving vehicles in tomorrow's smart cities mobility," $A I P$ Conference Proceedings, vol. 2040, no. 1, Article ID 140007, 2018.

[22] S. Wang, J. Yuan, X. Li, Z. Qian, F. Arena, and I. You, “Active data replica recovery for quality-assurance big data analysis in ic-iot," IEEE Access, vol. 7, pp. 106997-107005, 2019.

[23] F. Arena, G. Pau, and A. Severino, "V2x communications applied to safety of pedestrians and vehicles," Journal of Sensor and Actuator Networks, vol. 9, no. 1, 2020.

[24] F. Arena and G. Pau, "A review on ieee 802.11p for intelligent transportation systems," Journal of Sensor and Actuator Networks, vol. 9, no. 2, 2020.

[25] T. T. Almeida, L. De C. Gomes, F. M. Ortiz, J. R. Júnior, and L. H. M. K. Costa, "Ieee 802.11p performance evaluation: simulations vs. real experiments," in Proceedings of the 2018 21st International Conference On Intelligent Transportation Systems (ITSC), pp. 3840-3845, Maui, HI, USA, November, 2018.

[26] S. Benkirane and M. Benaziz, "Performance evaluation of ieee 802.11p and ieee 802.16e for vehicular ad hoc networks using simulation tools," in Proceedings of the 2018 IEEE 5th International Congress on Information Science and Technology (CiSt), pp. 573-577, Marrakech, Morocco, October, 2018.

[27] A. Bazzi, B. M. Masini, A. Zanella, and I. Thibault, "On the performance of ieee $802.11 \mathrm{p}$ and lte-v2v for the cooperative awareness of connected vehicles," IEEE Transactions on Vehicular Technology, vol. 66, no. 11, Article ID 10419, 2017.

[28] T. Gupta, F. Arena, and I. You, "Efficient resource allocation for backhaul-aware unmanned air vehicles-to-everything (u2x)," Sensors, vol. 20, p. 2994, 2020.

[29] X. Duan, Y. Yang, D. Tian, Y. Wang, and T. Li, “A v2x communication system and its performance evaluation test bed," in Proceedings of the 2014 IEEE 6th International Symposium on Wireless Vehicular Communications (WiVeC 2014), pp. 1-2, Vancouver, BC, Canada, September, 2014.

[30] F. Arena, G. Pau, and A. Severino, "An overview on the current status and future perspectives of smart cars," Infrastructure, vol. 5, no. 7, 2020.

[31] A. Sassi, F. Charfi, L. Kamoun, Y. Elhillali, and A. Rivenq, "The impact of mobility on the performance of $\mathrm{v} 2 \mathrm{x}$ communication," in Proceedings of the 2011 4th International Conference On Logistics, pp. 434-438, Hammamet Tunisia, June 2011. 
[32] N. Williams, G. Wu, K. Boriboonsomsin, M. Barth, S. Rajab, and S. Bai, "Anticipatory lane change warning using vehicleto-vehicle communications," in Proceedings of the 2018 21st International Conference On Intelligent Transportation Systems (ITSC), pp. 117-122, Maui, HI, USA, November, 2018.

[33] A. Cervin, D. Henriksson, B. Lincoln, J. Eker, and K.-E. Arzen, "How does control timing affect performance? analysis and simulation of timing using jitterbug and truetime," IEEE Control Systems Magazine, vol. 23, no. 3, pp. 16-30, 2003.

[34] M. K. Abbas, M. N. Karsiti, M. Napiah, B. B. Samir, and M. AlJemeli, "High accuracy traffic light controller for increasing the given green time utilization," Computers \& Electrical Engineering, vol. 41, pp. 40-51, 2015.

[35] L. Calderoni, D. Maio, and S. Rovis, "Deploying a network of smart cameras for traffic monitoring on a "city kernel"," Expert Systems with Applications, vol. 41, no. 2, pp. 502-507, 2014.

[36] N. I. Shuhaimi, Heriansyah, and T. Juhana, "Comparative performance evaluation of dsrc and wi-fi direct in vanet," in Proceedings of the 2015 4th International Conference On Instrumentation, Communications, Information Technology, and Biomedical Engineering (ICICI-BME), pp. 298-303, Bandung, Indonesia, November, 2015.

[37] D. Punia and R. Kumar, "Experimental characterization of routing protocols in urban vehicular communication," Transport and Telecommunication Journal, vol. 20, pp. 229-241, 2019.

[38] M. Balakrishnan and E. Johnson, "Queueing analysis of dchf and hf token protocol with varying turnaround time," Military Communications Conference, vol. 1, pp. 572-578, 2004. 